Article

\title{
Using Patent Technology Networks to Observe Neurocomputing Technology Hotspots and Development Trends
}

\author{
Shu-Hao Chang * ${ }^{(1)}$ and Chin-Yuan Fan \\ Science and Technology Policy Research and Information Center National Applied Research Laboratories, \\ Taipei 10636, Taiwan; cyfan@narlabs.org.tw \\ * Correspondence: shchang@narlabs.org.tw; Tel.: +866-2-27377779
}

Received: 20 July 2020; Accepted: 16 September 2020; Published: 17 September 2020

\begin{abstract}
In recent years, development in the fields of big data and artificial intelligence has given rise to interest among scholars in neurocomputing-related applications. Neurocomputing has relatively widespread applications because it is a critical technology in numerous fields. However, most studies on neurocomputing have focused on improving related algorithms or application fields; they have failed to highlight the main technology hotspots and development trends from a comprehensive viewpoint. To fill the research gap, this study adopts a new viewpoint and employs technological fields as its main subject. Neurocomputing patents are subjected to network analysis to construct a neurocomputing technology hotspot. The results reveal that the neurocomputing technology hotspots are algorithms, methods or devices for reading or recognizing printed or written characters or patterns, and digital storage characterized by the use of particular electric or magnetic storage elements. Furthermore, the technology hotspots are discovered to not be clustered around particular fields but, rather, are multidisciplinary. The applications that combine neurocomputing with digital storage are currently undergoing the most extensive development. Finally, patentee analysis reveal that neurocomputing technology is mainly being developed by information technology corporations, thereby indicating the market development potential of neurocomputing technology. This study constructs a technology hotspot network model to elucidate the trend in development of neurocomputing technology, and the findings may serve as a reference for industries planning to promote emerging technologies.
\end{abstract}

Keywords: neurocomputing; patent network; technology hotspot; network analysis

\section{Introduction}

Neurocomputing originally referred to hardware that mimics neuroscience structures to create models of the nervous system [1]. This concept is further extended to computing systems that operate using bioinspired computing models, including neural networks [2] and deep-learning networks [3]. In recent years, widespread research on neurocomputing technology has been driven by the rapid development of cognitive learning applications and the limited computing power of the Von Neumann architecture. In addition, the exponential development of big data and artificial intelligence (AI) has challenged the data processing speed and scalability of conventional computing systems. A report by $\mathrm{PwC}$ indicated that new computing technologies and the Internet of Everything are indispensable technologies in the march toward the fourth industrial revolution [4]. A Deloitte report even directly stated that companies' use of technologies related to neurocomputing, such as machine learning, is conducive to observing changes in customer population statistics [5]. Between 2019 and 2024, the fastest growing area in smart machine technology, namely neurocomputing, will grow at a $22.2 \%$ 
compound annual growth rate (CAGR) [6]. The changing face of Big Science is posing a crucial challenge to scholars. Critical topics include how human brain functions and consciousness can be recreated on supercomputers. Therefore, the development of technological platforms in the field of neurocomputing warrants further attention [7].

Research has mostly focused on improving neurocomputing methods [8-10] or application fields [11-13], whereas the hotspot fields required for the sustainable development of neurocomputing technology have been overlooked. In other words, research has not explained the current situations and positions of specific technological fields on a technology map or identified technology hotspots from a comprehensive perspective. Furthermore, development of neurocomputing technology involves numerous technological fields $[2,14,15]$, and such technology has limitless development potential. A hotspot refers to a study repeatedly cited in other different studies [16]. Namely, if one study frequently appears in other studies, it is a hotspot study. In the present study, the concept described by Mukherjee et al. [16] is used. A technology hotspot is defined as a technological field present in various patent documents. In other words, if multiple patents belong to the same technological field, then that field is a technology hotspot. The Cooperative Patent Classification (CPC) structure was used in this study to define a technological field. Research has indicated that, under most circumstances, the CPC structure is more detailed than that of the International Patent Classification (IPC) [17]. Namely, compared with IPC, CPC is a classification system with more detailed classifications and more additional texts.

Frost and Sullivan selected the top 50 emerging technologies from hundreds of technologies classified within nine technology clusters; their selections were based on industry adoption rate, internet protocol activity, funding, market potential, and whether the technology had the highest score on the global technology innovation index [18]. Convolutional neural network technology, a subset of the neurocomputing field, requires precise automatic processing, and numerous industries have been attracted to using the technology and investing in relevant research. Because neurocomputing offers limitless future business opportunities, defining the hotspots in the field is crucial. Research universities and operators yearn to determine the optimal resource allocation; namely, into which technological fields they should invest funding or their research workforce. To fill in the research gap, this study conducts technology hotspot network analysis to determine the current development conditions and positioning of particular technologies and to highlight the hotspot technological fields.

This study focuses on neurocomputing technology networks and employs patent analysis to construct a neurocomputing technology network model. Because patents are the most direct form of evaluation of innovation output, they serve as an indicator of trends in technological development [19-21]. Patent analysis methods are suitable for exploring technology transfer topics [22-24] and evaluating the performance of industry-academia technology collaborations [25,26]. Thus, patent information is the most direct evaluation indicator for technological development. The present study employs patent information to identify the trends in technological development and the critical fields in neurocomputing technology.

This study differs from other studies discussing the aspects and applications of neurocomputing technology because it focuses on neurocomputing technology hotspots, constructs a technology hotspot network model, and analyzes technological development trends. The technology hotspot network model uses networks to analyze the connections between different technology classification nodes to identify hotspot technologies. The principle is that one patent may be involved in multiple technological fields (technology nodes), and different patents may include overlapping technological fields. Therefore, similar to the relations among social members in a social network, a patent technological network comprises the relations among technology nodes. In addition, the network can be studied using social network analysis [27]. The findings of this study can serve as a reference for both academia and industry. 


\section{Literature Review}

\subsection{Current Development of Neurocomputing Technology}

Neurocomputing is a set of bioinspired computing paradigms and can be employed to solve certain problems in science and engineering, such as forecasting, pattern recognition, optimization, and identification of nonlinear systems. Neurocomputing applications learn through training processes and are divided into artificial neural networks (ANNs) and spiking neural networks (SNNs). Conventional ANNs are inspired by natural neural systems such as the brain. The human brain, for instance, consists of approximately $10^{11}$ neurons with more than $6 \times 10^{13}$ interconnections [28]. In general, computing is performed by neurons in a parallel and dispersed manner. This enables the human brain to demonstrate learning, recall, and generalization through sample or data training [29].

SNNs are a special class of ANNs in which communications occur through sequences of spikes [30]. First-generation ANNs comprise McCulloch-Pitts neurons, which can only obtain digital outputs; by contrast, in second-generation networks, neurons communicate continuously [28]. An SNN is a binary information transmission network. Neurons transmit pulses to subsequent neurons after receiving pulse signals that exceed a threshold potential. Neurons that have yet to receive pulses remain in the resting state. SNNs are thus highly tolerant of errors noise. Studies have revealed that the computing accuracy of synapses and neurons influences the final results of computations. Therefore, SNNs can be employed to solve problems for which only approximate answers are required, thereby conserving resources [31]. Scholars have also discovered that the computing costs of neural networks can be reduced using compression and sparse methods [32].

Neurocomputing has diverse applications and is used in numerous fields, including visual perception systems [33], communications [34], energy [35,36], and medicine [37]. The related literature is presented in Table 1. Neurocomputing topics include vision, signal and pattern processing, learning, neurodynamics, associative memory, and network hardware [38]. The rapid development of AI has caused greater demands for computational speed and resources. Neurocomputing networks can contain a large amount of information and imitate the brain's information processing. Therefore, industrial sectors worldwide highly value the development potential of neurocomputing technology and are allocating considerable resources to its development. The present study uses neurocomputing as its analysis subject and employs patent analysis to reveal the hotspots in the field of neurocomputing. Subsequently, these hotspot fields are explored by conducting network analysis. The analysis methods are explained in the following.

Table 1. Related literature on neurocomputing.

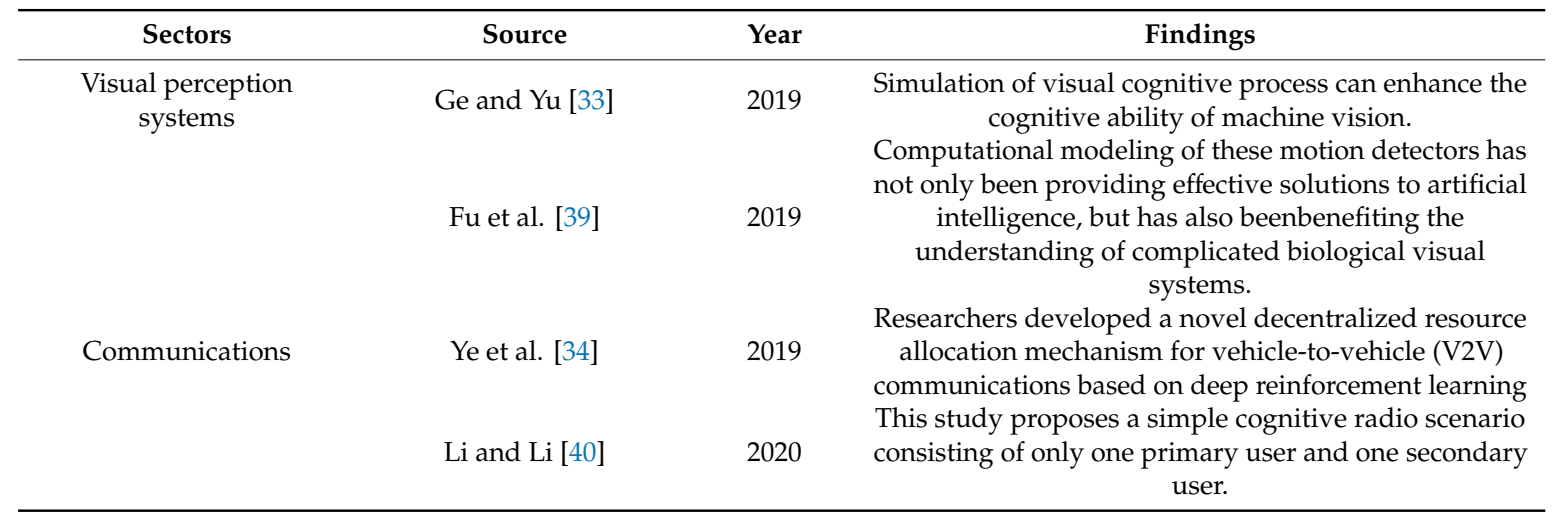


Table 1. Cont.

\begin{tabular}{|c|c|c|c|}
\hline Sectors & Source & Year & Findings \\
\hline Energy & Cao et al. [35] & 2015 & $\begin{array}{c}\text { Spiking neural networks (SNN)-based architectures } \\
\text { have shown great potential as a solution for realizing } \\
\text { ultra-low power consumption using spike-based } \\
\text { neuromorphic hardware. }\end{array}$ \\
\hline \multirow{3}{*}{ Medicine } & $\begin{array}{l}\text { Galindo Sanchez and } \\
\text { Nunez-Yanez [36] }\end{array}$ & 2017 & $\begin{array}{l}\text { This paper presents a high-performance architecture } \\
\text { for spiking neural networks that optimizes data } \\
\text { precision and streaming of configuration data stored in } \\
\text { main memory. }\end{array}$ \\
\hline & Lin et al. [37] & 2016 & $\begin{array}{l}\text { This survey makes an overview of the most recent } \\
\text { applications on the neural networks for } \\
\text { computer-aided medical diagnosis (CAMD) over the } \\
\text { past decade. }\end{array}$ \\
\hline & López-García et al. [41] & 2020 & $\begin{array}{l}\text { This paper presents pre-train convolutional neural } \\
\text { network architectures for survival prediction on a } \\
\text { subset composed of thousands of gene-expression } \\
\text { samples from thirty-one tumor types. }\end{array}$ \\
\hline
\end{tabular}

\subsection{Technology Hotspot Network Analysis}

Recent studies have used network analysis methods to explore the development of particular technologies [21,42,43], revealing the framework and scope of knowledge in the field [44,45] or employing patent analysis to determine the current conditions of technology transfer and cooperation $[23,24,46]$. Furthermore, network analysis can accurately identify the transfer channels and evolution of information technology. Patent analysis provides objective and accurate information, including the number of patents, approval year, and technology type [47]. Therefore, patent data analysis can meaningfully reveal the development of particular technologies. The present study employs network analysis to investigate the connectivity and co-occurrence between technology nodes. The technology node classification method proposed in Rodríguez-Salvador et al. [45] and Jürgens and Herrero-Solana [48] is used. By adopting a pre-existing and mature patent classification framework as its foundation, this study employs technology hotspot network analysis to identify the key players within the neurocomputing field, the neurocomputing hotspot fields, and the trends in neurocomputing development.

\section{Research Design}

\subsection{Search Strategy and Data Source}

Because the US patent system is generally representative of international technology and the United States is the largest commercial trading market, this study uses data from the United States Patent and Trademark Office (USPTO). Inventors commonly also apply for patents in the United States when applying for patents in other countries. Therefore, the USPTO database is commonly used to evaluate innovative activities worldwide $[42,43,49]$. The patents included in this study are limited to US patents announced between January 1990 and December 2019. According to research by Dehghani and Dangelico [50], the Derwent database is suitable. A precise search tool, namely, Derwent Smart Search, is employed for patent searching. The tool was designed by hundreds of experts who, after reading the officially announced patent data recorded in the Derwent database, translated, rewrote key abstracts, edited errors, and normalized the information on the patentee before uploading the rewritten and normalized data into the database. The search tool is used by inserting keywords established through manual reading and information organization. The search criteria of this study are as follows: (SSTO/neurocomputing) AND (SSTO/neural network). SSTO refers to Derwent Smart Search. In addition, patents in the CPC G06N3 class were targeted in this study. The most frequent G06N3 co-classifications were observed to track their development over time. In 2013, the USPTO and the European Patent Office jointly developed the CPC system. Kim and Bae [17] argued that CPC is a more detailed technology classification system than IPC. Therefore, this study employs the CPC 
system as the analysis framework. Finally, patents with US origins, which are purely domestic, were excluded to observe patents by patentees from countries outside the United States. In addition, the publication numbers of utility patents were used as the only identifiers for each patent to remove duplicate patents. A total of 635 patents were retrieved.

\subsection{Technology Hotspot Analysis}

Researchers have commonly used the network-centric analytic approach to reveal the hotspots within a network [51,52]. Because patents may be subordinated to numerous CPC categories, categories that share more patents have greater technological interrelatedness. Therefore, this study constructs a technology network comprising patents (internodes) between each CPC category (node). The key nodes situated in the center of the technology network are technology hotspots and nodes that have received the most interest. Social capital theory holds that the importance of each network node is determined by its centrality [53]. By adopting this theory, the present study reveals technology hotspots by calculating the network centrality of each node. The network centrality evaluation method is further explained as follows.

\subsubsection{Degree Centrality}

The neighborhood of a node is the nodes that are closest to it. The nodal degree of a node is the number of neighborhoods of that node and represents the number of relational lines that connect to the node. Degree of centrality is used to evaluate the core fields within the network. Nodes with a greater degree of centrality have more connections with other nodes within the network. Network nodes with a high degree of centrality serve as critical transition points and thus represent hotspots [54].

$$
\mathrm{C}_{d}(i)=\sum_{j} m_{j i}
$$

If nodes $i$ and $j$ are connected, $m_{j i}=1$.

\subsubsection{Eigenvector Centrality}

Eigenvector centrality measures the influence of a node in a network and determines whether the selected node is connected to numerous other nodes and whether those nodes are themselves connected to other nodes. Therefore, the centrality of the selected node determines the centrality of its neighborhoods. If the selected node is connected to nodes with high centrality, the selected node also has high centrality. Thus, the connections between neighborhoods are valued differently. Eigenvector centrality is calculated by assigning relative scores to all nodes in the network with the underlying idea that connections to high-scoring nodes should contribute more to the influence of the node than connections to low-scoring nodes [55]. Eigenvector centrality indicates the relative importance of a node and thus highlights the hotspots in the technology network.

$$
\mathrm{C}_{e}(i)=\lambda^{-1} \sum_{j=1}^{n} a_{i j} \mathrm{C}_{e}(j)
$$

where $C_{e}(i)$ is the eigenvector centrality of node $i, a_{i j}$ is the node that enters the adjacency matrix $\mathbf{A}$, and $\lambda$ is the maximum eigenvector value of the adjacency matrix, which is generally a common number.

Within the equation, the eigenvector centrality of the selected node is computed using a linear function in which the centrality of a single node is represented by the linear combination of the centrality of all other nodes [56]. 


\subsubsection{Betweenness Centrality}

Betweenness centrality means that some nodes in the network must rely on other nodes to connect with other nodes in the network. It can measure the importance of one node in data transmission. Nodes with higher betweenness centrality indicate that a technology has a crucial position in the network.

$$
\mathrm{C}_{b}(i)=\sum_{i \neq j \neq k}^{n} d_{j k}(i) / d_{j k}
$$

In Equation (3), $d_{j k}$ represents the number of shortest paths from node $j$ to node $k$, and $d_{j k}(i)$ represents the number of shortest paths that must pass through node $i$ from node $j$ to node $k$.

\section{Empirical Study}

\subsection{Patent Search Results}

Before technology network analysis is performed, the researchers analyze the patent search results to acquire initial understanding. Table 2 displays the 10 most common neurocomputing technology categories in the fourth hierarchical classification level of the CPC.

Table 2. Ten most common neurocomputing technology categories in the fourth hierarchical classification level of the Cooperative Patent Classification (CPC).

\begin{tabular}{cccc}
\hline Rank & CPC Code Number & Number of Occurrences & Percentage \\
\hline 1 & G06N3 & 635 & $40.60 \%$ \\
2 & G06K9 & 81 & $5.18 \%$ \\
3 & G06F9 & 62 & $3.96 \%$ \\
4 & G06F7 & 50 & $3.20 \%$ \\
5 & G11C11 & 50 & $3.20 \%$ \\
6 & G06F17 & 44 & $2.81 \%$ \\
7 & G11C13 & 32 & $2.05 \%$ \\
8 & G06F15 & 31 & $1.98 \%$ \\
9 & G06N20 & 31 & $1.98 \%$ \\
10 & G06T1 & 20 & $1.28 \%$ \\
\hline
\end{tabular}

This study analyzes the distribution of 162 neurocomputing technologies within the fourth hierarchical classification level of the CPC. In this study, the patents in the CPC G06N3 class were targeted, and the most frequent co-classifications of G06N3 were observed. Therefore, G06N3 was the primary technology. Table 2 indicates that neurocomputing technologies are mainly clustered around G06N3, G06K9, G06F9, G06F7, and G11C11. Each CPC category is defined in Appendix A.

According to the aforementioned analysis, the development of neurocomputing technology has focused on computer systems based on biological models (G06N3), methods or arrangements for reading or recognizing printed or written characters or for recognizing patterns (G06K9), arrangements for program control (G06F9), methods or arrangements for processing data by operating upon the order or content of the data handled (G06F7), and digital stores characterized by the use of particular electric or magnetic storage elements (G11C11). Table 3 presents the results of analysis of the 10 most prolific patentees. IBM (Armonk, NY, USA), which had the most patents, is devoted to developing AI, including the Neural Network Synthesizer (NeuNetS). NeuNetS can automatically synthesize and define neural networks and thus can be employed to accelerate the development of deep-learning network models. NeuNetS can optimize the processing speed or preciseness of a model and provide timely surveillance of the model construction and training process. The most prolific patentees after IBM (Armonk, NY, USA) are Google Inc. (Mountain View, CA, USA) and Intel Corporation (Santa Clara, US), technology giants that focus on developing AI and the Internet of Things (IoT) and endeavor 
to integrate artificial IoT solutions for consumers, enterprises, and industries across a wide variety of industries [57].

Table 3. Most prolific patentees.

\begin{tabular}{cccc}
\hline Rank & Patentee & Number of Patents & Percentage \\
\hline 1 & International Business Machines & 158 & $24.20 \%$ \\
2 & Corporation & 46 & $7.04 \%$ \\
3 & Google Inc. & 44 & $6.74 \%$ \\
4 & Intel Corporation & 24 & $3.68 \%$ \\
5 & Brain Corporation & 23 & $3.52 \%$ \\
6 & Via Alliance Semiconductor Co., Ltd. & 20 & $3.06 \%$ \\
7 & Motorola, Inc. & 19 & $2.91 \%$ \\
8 & Samsung Electronics Co., Ltd. & 18 & $2.76 \%$ \\
9 & Mitsubishi Denki Kabushiki Kaisha & 14 & $2.14 \%$ \\
10 & Hitachi, Ltd. & 11 & $1.68 \%$ \\
\hline
\end{tabular}

Note: For some of the American companies' patents, the patent application was proposed by a foreign subsidiary and was certified.

\subsection{Technology Hotspot Network Analysis}

This study uses the fourth hierarchical classification level of the CPC as a foundation for identifying patent technology hotspots. Figure 1 displays the technology hotspot network model, and Table 4 lists the CPC codes of each hotspot.

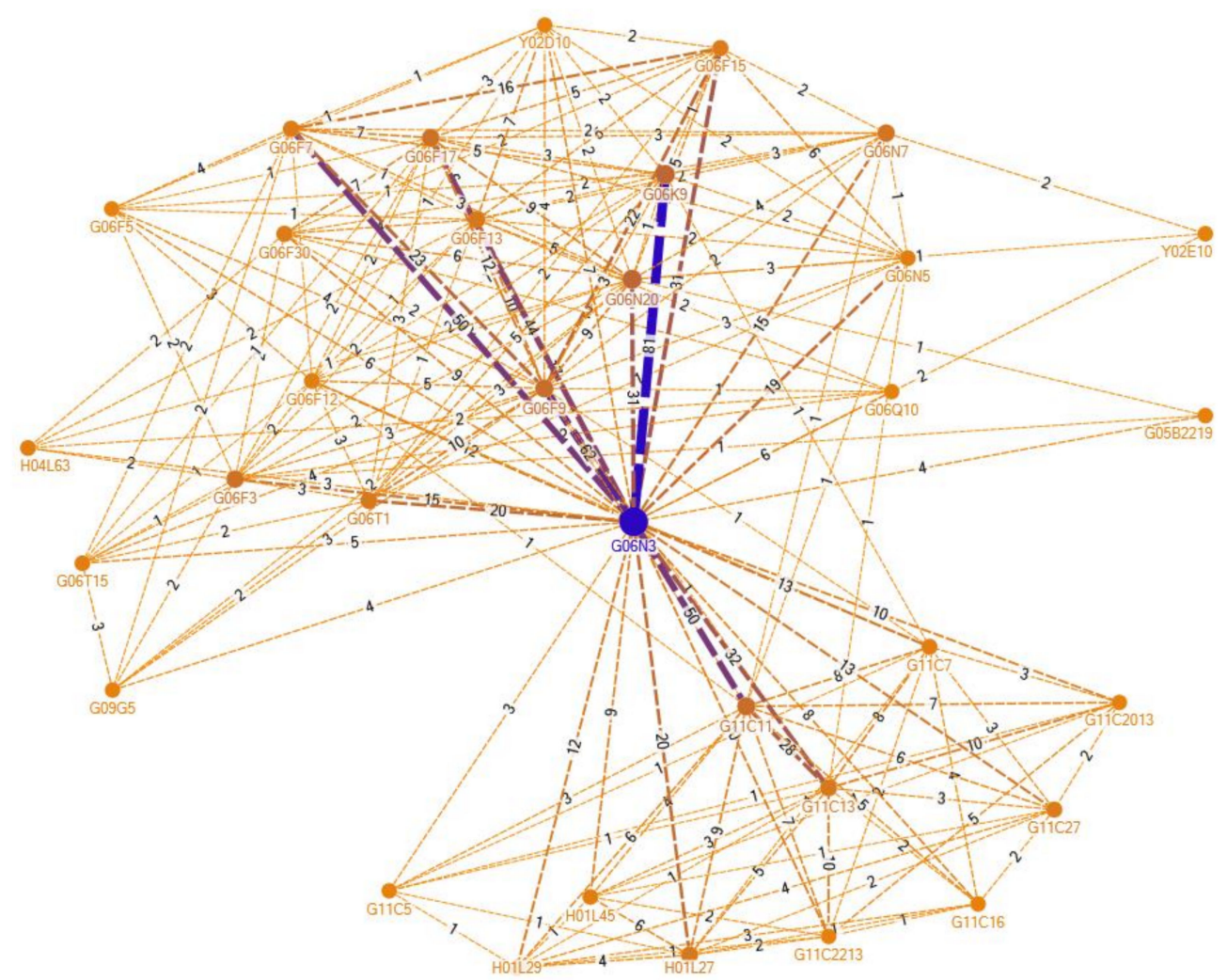

Figure 1. Neurocomputing technology network in the fourth hierarchical classification level of the CPC. Node size represents the number of nodes that are connected to the node. Line thickness represents the connectivity strength between each node and thus the number of shared patents. For simplicity, the figure only displays the nodes that are connected to more than 10 other nodes. 
Table 4. CPC codes of the five largest neurocomputing technology hotspots.

\begin{tabular}{cccccc}
\hline CPC & $\begin{array}{c}\text { Degree } \\
\text { Centrality }\end{array}$ & CPC & $\begin{array}{c}\text { Eigenvector } \\
\text { Centrality }\end{array}$ & CPC & $\begin{array}{c}\text { Betweenness } \\
\text { Centrality }\end{array}$ \\
\hline G06N3 & 162 & G06N3 & 0.480 & G06N3 & $10,576.56$ \\
G06N20 & 45 & G06N20 & 0.210 & G06K9 & 330.873 \\
G06K9 & 44 & G06F9 & 0.206 & G06N20 & 309.304 \\
G06F9 & 36 & G06K9 & 0.197 & G11C11 & 165.425 \\
G11C11 & 36 & G06F17 & 0.182 & G06F9 & 124.946 \\
\hline
\end{tabular}

Table 4 indicates that G06N3, G06N20, G06K9, G06F9, and G11C11 were ranked within the top five in two or more of the three centrality indexes. Thus, within neurocomputing technology hotspots, the main technologies are computer systems based on biological models (G06N3), machine learning (G06N20), methods or arrangements for reading or recognizing printed or written characters or patterns (e.g., fingerprints; G06K9), arrangements for program control, e.g., control units (G06F9), and digital stores characterized by the use of particular electric or magnetic storage elements (G11C11).

\subsection{Postanalysis: History of Neurocomputing Technology Hotspots and Clustering Analysis}

This study conducts further analysis on the history of G06N3, G06N20, G06K9, G06F9, and G11C11 to understand the trend in neurocomputing technology. The analysis results are displayed in Figure 2.

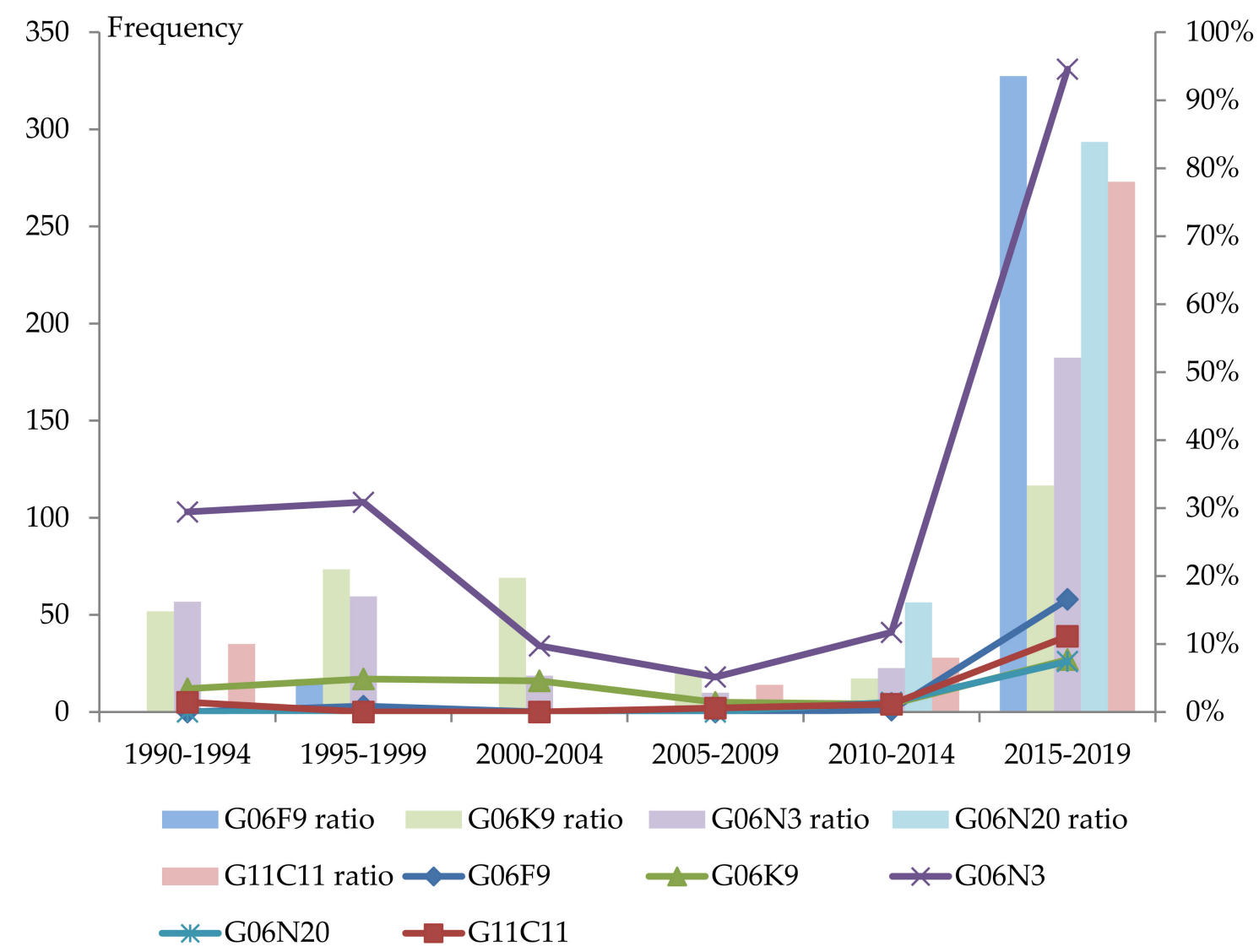

Figure 2. History of neurocomputing technology hotspots. The bars represent the ratios of the numbers of patents in a particular year among all patents. The lines represent the number of patents.

Figure 2 suggests that, before 2010, development within neurocomputing was mainly focused on G06N3; the development of mathematical models. After 2010, neurocomputing applications were 
employed in other fields. This is particularly evident after 2014. In addition, the development of G11C11, namely neurocomputing applications in digital-store-related technologies, warrants attention. This development was caused by increasing demand for storage in big data computing, in which digital memory storage applications play a critical role. The relative development of G11C11-related technologies and patents has thus increased in recent years. For the distribution of ratios, a larger ratio of G06K9 patents, compared with patents in other technological fields, was approved before 2014. This indicates that neurocomputing has a longer history of application in pattern recognition (G06K9).

Research has indicated that patent clustering analysis in network analysis can provide management implications [58]. In this study, clustering analysis was conducted with factionalization to obtain more information. The results are presented in Table 5.

Table 5. Cluster Interpretations.

\begin{tabular}{cll}
\hline Cluster & \multicolumn{1}{c}{ Main CPC Classes in the Cluster } & \multicolumn{1}{c}{ Interpretation } \\
\hline 1 & G05B13, G06G7, G16H50, G02F1 & $\begin{array}{l}\text { Adaptive control systems and interdisciplinary } \\
\text { applications, such as optics and information and } \\
\text { communications technology (ICT) specially adapted } \\
\text { for medical diagnosis }\end{array}$ \\
2 & G11C7, G11C11, G11C13, H01L21, H01L27 & $\begin{array}{l}\text { Digital storage elements and semiconductor devices } \\
\text { Recognizing patterns, image analysis, and robots }\end{array}$ \\
3 & G06K9, G06T2207, G06T5, Y10S901, B25J9 & $\begin{array}{l}\text { Electric digital data processing and specific } \\
\text { computational models }\end{array}$ \\
\hline
\end{tabular}

Factions is an algorithm that can be used to search for the optimal method of assigning actors in factions with a total of four small groups. The E-I index was -0.437 , and the negative value indicated that the degree of segmentation among clusters was high. In addition, the correct final proportion was 0.780, which indicated that the clustering results already had favorable fit values. Table 5 indicates that patents for neurocomputing technology are distributed in several major clusters, including interdisciplinary applications such as optics and medical information communications technology (ICT), digital storage equipment and semiconductors, pattern recognition and robot development, and specific computational models.

\section{Conclusions}

\subsection{Discussion}

This study employs network analysis to explore hotspots in the field of neurocomputing technology. The empirical research results indicate that rather than concentrating on a particular field, innovators have focused on algorithms, machine learning, methods or devices for recognizing patterns, arrangements for program control, and electric- or magnetic-based digital storage. This indicates that neurocomputing is a field of multidisciplinary technological development. In addition, through network analysis and by comparing the most frequent co-classifications of G06N3, we learned that G06N3, G06K9, G06F9, and G11C11 ranked in the top five in the network and co-classification analyses. However, although G06N20 was ranked among the top five in the network analysis, its frequency did not reach the top five in the co-classification analysis. Therefore, through network analysis, we gained the additional insight that, although machine learning (G06N60) exhibited a relatively low frequency in patents, in the overall technology network, the technology nodes it connected were diverse and exhibited characteristics of interdisciplinary application. For example, the degree centrality of G06N60 demonstrated that it was connected to many nodes. Eigenvector centrality showed whether G06N20 was connected to nodes with high centrality. Betweenness centrality revealed that G06N20 occupied critical channels in network communication, reflecting the degree to which connections among technology sets rely on G06N20. Patentee analysis further revealed that IBM (Armonk, NY, US) and Qualcomm Inc. (San Diego, CA, USA) obtained the most patents in the study period. Therefore, development in the field of neurocomputing is mainly being conducted by technology giants that are 
developing AI and the IoT, and neurocomputing technology has the potential for development in future markets. Driven by anticipated market profit, private-sector corporations have begun active research and development of neurocomputing technology while planning to introduce commercial neurocomputing technology applications to the market in the near future.

This study also reveals that, in addition to G06N3 (computer systems based on biological models), G11C11 (digital stores characterized by the use of particular electric or magnetic storage elements) is also undergoing considerable development. Therefore, applications that combine neurocomputing technology with digital storage are a hotspot in the future development of big data. Inspired by the neuron and synaptic mechanism theory of the human brain, scientists have developed AI computing chips that can provide timely computing to satisfy the demand for smart devices [59]. In 2018, the AI chip market was valued at US $\$ 6638$ million. By 2025, it is expected to reach US\$91,185 million, a compound annual growth rate of $45.2 \%$ from 2019 to 2025 [60]. Additionally, digital storage technology for neuromorphic chips, which are components that simulate neuron cells (e.g., memristors) [61], is a relatively popular neurocomputing field that has attracted considerable attention in recent years. In addition, from the perspective of causality in technology sequences, in the early period, biological models (G06N3) with high-technology characteristics were often applied in application fields such as pattern recognition methods (G06K9). In recent years, technologies and related applications have appeared that require large amounts of computing, such as machine learning (G06N20) with high-technology characteristics and neurocomputing applications in digital-store-related technologies (G11C11) with high-application characteristics. In the aforementioned causality analysis, we learned that as communications technology and big data have developed, in addition to early applications in biometric identification, digital storage memory and smart computing have gradually attracted attention and led to more technological development and relevant applications.

From the theoretical contribution viewpoint, studies conducted in the neurocomputing field have focused on neurocomputing technology improvements [8-10] or applications [11-13]. However, these studies failed to highlight the key hotspots, development trends, and network distribution and context of the neurocomputing field. Neurocomputing is a core technology in numerous technology-related fields. To fill in the research gap, this study uses a novel perspective to identify the critical fields in neurocomputing.

\subsection{Industrial Implications}

This study provides researchers in industry and academia with valuable information and proposes a technology map of the neurocomputing field. This map highlights the key technology developments in the neurocomputing field and finds answers to resource allocation problems. This study also reveals that neurocomputing, combined with digital storage technology, is a hotspot field. In the age of big data and AI, everyday appliances (e.g., IoT devices, cars, and cellphones) will be connected to the internet, generating big data and increasing the demand for storage devices with greater capacity and memory. One possible solution to the digital storage problem is to replace integrated circuits with neuromorphic computer systems. Such systems comprise numerous nodes, each representing a neuron. Information is transferred between nodes, with each node demonstrating the ability to compute data. Neuromorphic computer systems thus have processing-in-memory functionality and can compute and store information within each "neuron." This technology involves numerous fields, including computer information, communications, mathematics, neurobiology, and cognitive science. Because pilot studies typically require substantial equipment and investment in expert personnel, industry-academia cooperation should lead the academic field by investing in neurocomputing-related basic and applied research, establishing goals to develop practical applications, making long-term investments, and cultivating relevant talent to respond to industry demands for the future age of AI. Specifically, neural networks are part of the broader field of AI. AI studies can be divided into two main schools; namely, numerical AI and symbolic AI. Symbolic AI can be considered classical AI, which primarily manages various problems in human life related to symbols. Currently, numerical 
AI uses data to express a problem, and its development is supported by major technologies such as machine learning. On the basis of the aforementioned description, cognitive learning applications and numerical calculations in AI are highly related to pattern recognition, machine learning, and computer systems based on biological models in neural networks. The technological development of neural networks is key in AI.

In addition, the patent clustering analysis in this study indicated that most patents for neurocomputing technology were in optics, ICT and medicine, pattern recognition, and robot development, as well as in the development of specific computational models. With the development of the Internet of Things and big data techniques, optical communication and related uses (such as remote medical diagnosis) are gradually increasing in value. Research by MarketsandMarkets indicated that the size of the market global optical communication and networking equipment will be US $\$ 18.9$ billion in 2020. However, it will grow to US $\$ 27.8$ billion by 2025 , and its CAGR will be $8.0 \%$ [62]. In the field of optical communications, a combination of math, programming, and algorithms related to neurocomputing technology is required. This is also a crucial development direction for future neurocomputing technologies. In addition, the analysis in this study demonstrated that pattern recognition and robots are also a key development direction for neurocomputing technology. Generally, training robots to perform round trajectory tracing is more complicated and difficult compared with straight-line tracing. The improvement of robot control and signal processing models will become a main stream of business research in the future.

\subsection{Limitations and Future Research Directions}

This study exclusively employs patents to investigate trends in technological development. Although patents reflect the development dynamics of commercial technologies, neurocomputing development is reflected by numerous different forms in addition to patents, including theses, technology market reports, and other particular products. However, these forms were not within the scope of this study, serving as a major limitation. This study is a quantitative study and considers large-scale and comprehensive neurocomputing technology networks. In an attempt to encompass wide-ranging research topics, this study could obtain limited insight on each research topic. Future studies should analyze the content of all patents and evaluate their uniqueness. Finally, this study exclusively employs the database of the largest commercial trade market in the world, namely the USPTO database, as the source of patent information due to limited resources and funding. Although the database used in this study has a certain history and representativeness [42,43,49], future researchers should include observations and verifications using information obtained from other patent bureaus (e.g., the Worldwide Patent Statistical Database of the European Patent Office or the database of the Japan Patent Office) to expand the research scope.

Author Contributions: S.-H.C. contributed to the following: the conception and design of the study, acquisition of data, analysis, and interpretation of data, drafting the article and approval of the submitted version. Moreover, C.-Y.F. contributed to providing assistance in the construction of the patent database. All authors have read and agreed to the published version of the manuscript.

Funding: This research was funded by the Ministry of Science and Technology of Taiwan, grant number MOST 109-2410-H-492-001.

Acknowledgments: The authors would like to thank the Ministry of Science and Technology of Taiwan for financially supporting this research under Contract No. MOST 109-2410-H-492-001.

Conflicts of Interest: The authors declare no conflict of interest. 


\section{Appendix A}

Table A1. Definition of CPC categories in the fourth hierarchical classification level.

\begin{tabular}{|c|c|}
\hline CPC Categories & Meaning \\
\hline B25J9 & Program-controlled manipulators \\
\hline G02F1 & $\begin{array}{l}\text { Devices or arrangements for the control of the intensity, color, phase, polarization, or } \\
\text { direction of light arriving from an independent light source, e.g., switching, gating, or } \\
\text { modulating; Non-linear optics }\end{array}$ \\
\hline G05B13 & $\begin{array}{l}\text { Adaptive control systems, i.e., systems automatically adjusting themselves to have a } \\
\text { performance that is optimum according to some preassigned criterion }\end{array}$ \\
\hline G06F7 & $\begin{array}{l}\text { Methods or arrangements for processing data by operating upon the order or content of the } \\
\text { data handled }\end{array}$ \\
\hline G06F9 & Arrangements for program control, e.g., control units \\
\hline G06F15 & Digital computers in general; Data processing equipment in general \\
\hline G06F17 & $\begin{array}{l}\text { Digital computing or data processing equipment or methods, specially adapted for specific } \\
\text { functions }\end{array}$ \\
\hline G06G7 & $\begin{array}{l}\text { Devices in which the computing operation is performed by varying electric or magnetic } \\
\text { quantities }\end{array}$ \\
\hline G06K9 & $\begin{array}{l}\text { Methods or arrangements for reading or recognizing printed or written characters or for } \\
\text { recognizing patterns, e.g., fingerprints }\end{array}$ \\
\hline G06N3 & Computer systems based on biological models \\
\hline G06N20 & Machine learning \\
\hline G06T1 & General purpose image data processing \\
\hline G06T2207 & Indexing scheme for image analysis or image enhancement \\
\hline G06T5 & Image enhancement or restoration \\
\hline G11C7 & Arrangements for writing information into, or reading information out of, a digital store \\
\hline G11C11 & $\begin{array}{l}\text { Digital stores characterized by the use of particular electric or magnetic storage elements; } \\
\text { Storage elements thereof }\end{array}$ \\
\hline G11C13 & Digital stores characterized by the use of storage elements not covered by groups \\
\hline G16H50 & $\begin{array}{l}\text { ICT specially adapted for medical diagnosis, medical simulation, or medical data mining; } \\
\text { ICT specially adapted for detecting, monitoring, or modelling epidemics or pandemics }\end{array}$ \\
\hline H01L21 & $\begin{array}{l}\text { Processes or apparatus adapted for the manufacture or treatment of semiconductor or solid } \\
\text { state devices or of parts thereof }\end{array}$ \\
\hline H01L27 & $\begin{array}{l}\text { Devices consisting of a plurality of semiconductor or other solid-state components formed in } \\
\text { or on a common substrate }\end{array}$ \\
\hline Y10S901 & Robots \\
\hline
\end{tabular}

\section{References}

1. Mead, C. Analog VLSI and Neural Systems; Addison-Wesley Longman Publishing: Boston, MA, USA, 1989.

2. Serrano, W.; Gelenbe, E. The random neural network in a neurocomputing application for Web search. Neurocomputing 2018, 280, 123-134. [CrossRef]

3. Langenkämper, D.; Simon-Lledó, E.; Hosking, B.; Jones, D.O.B.; Nattkemper, T.W. On the impact of citizen science-derived data quality on deep learning based classification in marine images. PLoS ONE 2019, 14, e0218086.

4. PWC. Fourth Industrial Revolution for the Earth; PWC Publishers: London, UK, 2017.

5. Deloitte. Cognitive Intelligence; Deloitte Publishers: London, UK, 2016.

6. Research and Markets. Artificial Intelligence, Industrial Iot, and Smart Machines in Enterprise E Industrial Automation (2019-2024); Research and Markets: Dublin, Ireland, 2019.

7. Ulmi, N. Large-scale research: From atomic bombs to citizen science. Horizons-The Swiss Research Magazine. Available online: https://www.horizons-mag.ch/2018/12/06/large-scale-research-from-atomicbombs-to-citizen-science/ (accessed on 12 January 2020).

8. Huang, D.S.; Premaratne, P. Advanced neurocomputing theory and methodology. Neurocomputing 2007, 70, 623-624. [CrossRef]

9. Li, H.; Duan, W.; Huang, H.Z. Neurocomputing method based on structural finite element analysis of discrete model. Neural Comput. Appl. 2010, 19, 875-882. [CrossRef]

10. Pedrycz, W.; Aliev, R.A. Logic-oriented neural networks for fuzzy neurocomputing. Neurocomputing 2009, 73, 10-23. [CrossRef] 
11. Belayadi, A.; Bourahla, B.; Mekideche-Chafa, F. Neurocomputing techniques to predict the 2D structures by using lattice dynamics of surfaces. Acta Phys. Pol. 2017, 132, 1314-1319. [CrossRef]

12. Demertzis, K.; Iliadis, L. Detecting invasive species with a bio-inspired semi-supervised neurocomputing approach: The case of Lagocephalus sceleratus. Neural Comput. Appl. 2017, 28, 1225-1234. [CrossRef]

13. Pratama, M.; Lughofer, E.; Wang, D. Online real-time learning strategies for data streams for Neurocomputing. Neurocomputing 2017, 262, 1-3. [CrossRef]

14. Srinivasan, S.; Saghir, M. A neurocomputing model to calculate the thermo-solutal diffusion in liquid hydrocarbon mixtures. Neural Comput. Appl. 2014, 24, 287-299. [CrossRef]

15. Serpen, G.; Liu, L. Parallel and distributed neurocomputing with wireless sensor networks. Neurocomputing 2016, 173, 1169-1182. [CrossRef]

16. Mukherjee, S.; Romero, D.M.; Jones, B.; Uzzi, B. The nearly universal link between the age of past knowledge and tomorrow's breakthroughs in science and technology: The hotspot. Sci. Adv. 2017, 3, e1601315. [CrossRef] [PubMed]

17. Kim, G.; Bae, J. A novel approach to forecast promising technology through patent analysis. Technol. Forecast. Soc. Chang. 2017, 117, 228-237. [CrossRef]

18. Kumar, R. Top 50 Emerging Technologies \& Growth Opportunities; Frost \& Sullivan: San Antonio, TX, USA, 2019.

19. Gui, B.; Ju, Y.; Liu, Y. Mapping technological development using patent citation trees: An analysis of bogie technology. Technol. Anal. Strateg. Manag. 2019, 31, 213-226. [CrossRef]

20. Woo, H.G.; Yeom, J.; Lee, C. Screening early stage ideas in technology development processes: A text mining and K-nearest neighbours approach using patent information. Technol. Anal. Strateg. Manag. 2019, 31, 532-545. [CrossRef]

21. You, H.; Li, M.; Hipel, K.; Jiang, J.; Ge, B.; Duan, H. Development trend forecasting for coherent light generator technology based on patent citation network analysis. Scientometrics 2017, 111, 297-315. [CrossRef]

22. Donges, A.; Selgert, F. Technology transfer via foreign patents in Germany, 1843-1877. Econ. Hist. Rev. 2019, 72, 182-208. [CrossRef]

23. Nordensvard, J.; Zhou, Y.; Zhang, X. Innovation core, innovation semi-periphery and technology transfer: The case of wind energy patents. Energy Policy 2018, 120, 213-227. [CrossRef]

24. Seo, I.; Sonn, J.W. The persistence of inter-regional hierarchy in technology transfer networks: An analysis of Chinese patent licensing data. Growth Chang. 2019, 50, 145-163. [CrossRef]

25. Ardito, L. Markets for university inventions: The role of patents' underlying knowledge in university-to-industry technology commercialization. Int. J. Technol. Manag. 2018, 78, 9-27. [CrossRef]

26. MingJi, J.; Ping, Z. Research on the patent innovation performance of university-industry collaboration based on complex network analysis. J. Bus. Bus. Mark. 2014, 21, 65-83. [CrossRef]

27. Choi, J.Y.; Jeong, S.; Jung, J.K. Evolution of technology convergence networks in Korea: Characteristics of temporal changes in R\&D according to institution type. PLOS ONE 2018, 13, e0192195.

28. Ahmadi, A. Evolving spiking neural networks for control of artificial creatures. Brain Broad Res. Artif. Intell. Neurosci. 2013, 4, 5-19.

29. Watson, R.A.; Mills, R.; Buckley, C.L. Global adaptation in networks of selfish components: Emergent associative memory at the system scale. Artif. Life 2011, 17, 147-166. [CrossRef]

30. Ponulak, F.; Kasinski, A. Introduction to spiking neural networks: Information processing, learning and applications. Acta Neurobiol. Exp. 2011, 71, 409-433.

31. Sen, S.; Venkataramani, S.; Raghunathan, A. Approximate computing for spiking neural networks. In Proceedings of the Design, Automation \& Test in Europe Conference Exhibition (DATE), Lausanne, Switzerland, 27-31 March 2017; IEEE; pp. 193-198.

32. Han, S.; Mao, H.; Dally, W.J. Deep compression: Compressing deep neural networks with pruning, trained quantization and Huffman coding. In Proceedings of the International Conference on Learning Representations, ICLR, San Juan, Puerto Rico, 2-4 May 2016; pp. 1-14.

33. Ge, H.; Yu, H. The application and design of neural computation in visual perception. J. Vis. Commun. Image Represent. 2019, 59, 309-315. [CrossRef]

34. Ye, H.; Li, G.Y.; Juang, B.H.F. Deep reinforcement learning based resource allocation for V2V communications. IEEE Trans. Veh. Technol. 2019, 68, 3163-3173. [CrossRef]

35. Cao, Y.; Chen, Y.; Khosla, D. Spiking deep convolutional neural networks for energy-efficient object recognition. Int. J. Comput. Vis. 2015, 113, 54-66. [CrossRef] 
36. Galindo Sanchez, F.; Nunez-Yanez, J. Energy proportional streaming spiking neural network in a reconfigurable system. Microprocess. Microsyst. 2017, 53, 57-67. [CrossRef]

37. Lin, D.; Vasilakos, A.V.; Tang, Y.; Yao, Y. Neural networks for computer-aided diagnosis in medicine: A review. Neurocomputing 2016, 216, 700-708. [CrossRef]

38. Kang, G.B.; Baidyk, T.; Blanco, A.; Chan, T.W.; Chan, K.F. Progress in Eurocomputing Research; Nova Science Publishers: New York, NY, USA, 2008.

39. Fu, Q.; Wang, H.; Hu, C.; Yue, S. Towards computational models and applications of insect visual systems for motion perception: A review. Artif. Life 2019, 25, 263-311. [CrossRef]

40. Li, M.; Li, H. Application of deep neural network and deep reinforcement learning in wireless communication. PLoS ONE 2020, 15, e0235447. [CrossRef] [PubMed]

41. López-García, G.; Jerez, J.M.; Franco, L.; Veredas, F.J. Transfer learning with convolutional neural networks for cancer survival prediction using gene-expression data. PLOS ONE 2020, 15, e0230536.

42. Huang, C.; Lin, H.H.; Wan, J.B.; He, C.; Hu, Y. Research and development of hepatitis B drugs: An analysis based on technology flows measured by patent citations. PLOS ONE 2016, 11, e0164328. [CrossRef]

43. van den Oord, A.; van Witteloostuijn, A. A multi-level model of emerging technology: An empirical study of the evolution of biotechnology from 1976 to 2003. PLoS ONE 2018, 13, e0197024. [CrossRef] [PubMed]

44. Khan, G.; Wood, J. Information technology management domain: Emerging themes and keyword analysis. Scientometrics 2015, 105, 959-972. [CrossRef]

45. Rodríguez-Salvador, M.; Rio-Belver, R.M.; Garechana-Anacabe, G. Scientometric and patentometric analyses to determine the knowledge landscape in innovative technologies: The case of 3D bioprinting. PLoS ONE 2017, 12, e0180375.

46. de Paulo, A.F.; Ribeiro, E.M.S.; Porto, G.S. Mapping countries cooperation networks in photovoltaic technology development based on patent analysis. Scientometrics 2018, 117, 667-686. [CrossRef]

47. Leydesdorff, L.; Kogler, D.; Yan, B. Mapping patent classifications: Portfolio and statistical analysis, and the comparison of strengths and weaknesses. Scientometrics 2017, 112, 1573-1591. [CrossRef]

48. Jürgens, B.; Herrero-Solana, V. Monitoring nanotechnology using patent classifications: An overview and comparison of nanotechnology classification schemes. J. Nanoparticle Res. 2017, 19, 1-7. [CrossRef]

49. Bass, S.D.; Kurgan, L.A. Discovery of factors influencing patent value based on machine learning in patents in the field of nanotechnology. Scientometrics 2010, 82, 217-241. [CrossRef]

50. Dehghani, M.; Dangelico, R.M. Smart wearable technologies: State of the art and evolution over time through patent analysis and clustering. Int. J. Prod. Dev. 2018, 22, 293-313. [CrossRef]

51. Fang, Y. Visualizing the structure and the evolving of digital medicine: A scientometrics review. Scientometrics 2015, 105, 5-21. [CrossRef]

52. Zhao, R.; Wei, M.; Quan, W. Evolution of think tanks studies in view of a scientometrics perspective. Knowl. Organ. 2017, 44, 335-348. [CrossRef]

53. Saffer, A.J. A message-focused measurement of the communication dimension of social capital: Revealing shared meaning in a network of relationships. J. Public Relat. Res. 2016, 28, 170-192. [CrossRef]

54. Leydesdorff, L.; Nooy, W. Can 'hot spots' in the sciences be mapped using the dynamics of aggregated journal-journal citation relations? J. Assoc. Inf. Sci. Technol. 2017, 68, 197-213. [CrossRef]

55. Batool, K.; Niazi, M.A. Towards a methodology for validation of centrality measures in complex networks. PLoS ONE 2014, 9, e90283. [CrossRef]

56. Borgatti, S.P.; Everett, M.G.A. Graph-theoretic perspective on centrality. Soc. Netw. 2006, 28, 466-484. [CrossRef]

57. Research and Markets. Artificial Intelligence and Internet of Things Convergence: Aiot Technologies, Integration, Infrastructure, Solutions, Applications and Services by Industry Vertical 2018-2023; Research and Markets: Dublin, Ireland, 2018.

58. Dehghani, M.; Mashatan, A.; Kennedy, R.W. Innovation within networks-patent strategies for blockchain technology. J. Bus. Ind. Mark. 2020; ahead-of-print.

59. Monroe, D. Chips for artificial intelligence: Companies are racing to develop hardware that more directly empowers deep learning. Commun. ACM 2018, 61, 15-17. [CrossRef]

60. Rahul, K.; Supradip, B. Artificial Intelligence Chip Market; Allied Market Research: Portland, OR, USA, 2019. 
61. Zeng, X.; Wen, S.; Zeng, Z.; Huang, T. Design of memristor-based image convolution calculation in convolutional neural network. Neural Comput. Appl. 2018, 30, 503-508. [CrossRef]

62. MarketsandMarkets. Optical Communication and Networking Equipment Market by Component (Fiber, Transceiver, and Switch), Technology, Application (Telecom, Data Center, and Enterprise), Data Rate, Vertical, and Region-Global Forecast to 2025; MarketsandMarkets: Northbrook, IL, USA, 2020. 